\title{
Erratum to: Globalisation Sets the Background to the Crisis
}

\section{Erratum to:}

Chapter 1 in: A. Blundell-Wignall et al., Globalisation and Finance at the Crossroads, https://doi.org/10.1007/978-3-319-72676-2_1

The original version of the book was inadvertently published with misspelt author name "Blundell-Wignal et al." in the opening page footnote of Chapter 1, which has to be now corrected to read as "BlundellWignall et al.". The erratum chapter and the book have been updated with the change. 